\title{
Ultrafast and high-throughput mass spectrometric assay for therapeutic drug monitoring of antiretroviral drugs in pediatric HIV-1 infection applying dried blood spots
}

\author{
Roland J. W. Meesters • Jeroen J. A. van Kampen • Mariska L. Reedijk • \\ Rachel D. Scheuer • Lennard J. M. Dekker • David M. Burger • Nico G. Hartwig • \\ Albert D. M. E. Osterhaus • Theo M. Luider • Rob A. Gruters
}

Received: 28 April 2010 /Revised: 18 June 2010 /Accepted: 20 June 2010 /Published online: 15 July 2010

(C) The Author(s) 2010. This article is published with open access at Springerlink.com

\begin{abstract}
Kaletra ${ }^{\circledR}$ (Abott Laboratories) is a co-formulated medication used in the treatment of HIV-1-infected children, and it contains the two antiretroviral protease inhibitor drugs lopinavir and ritonavir. We validated two new ultrafast and high-throughput mass spectrometric assays to be used for therapeutic drug monitoring of lopinavir and ritonavir concentrations in whole blood and in plasma from HIV-1-infected children. Whole blood was blotted onto dried blood spot (DBS) collecting cards, and plasma was collected simultaneously. DBS collecting cards were extracted by an acetonitrile/water mixture while plasma samples were deproteinized with acetone. Drug concen-
\end{abstract}

R. J. W. Meesters $(\bowtie) \cdot$ J. J. A. van Kampen · L. J. M. Dekker •

T. M. Luider

Department of Neurology, Laboratory of Neuro-Oncology

and Clinical and Cancer Proteomics, University Medical Center

Rotterdam (Erasmus MC),

Dr. Molewaterplein 50, Room Ee-1981,

3015 GE Rotterdam, The Netherlands

e-mail: r.meesters@erasmusmc.nl

J. J. A. van Kampen • M. L. Reedijk • R. D. Scheuer ·

A. D. M. E. Osterhaus $\cdot$ R. A. Gruters

Department of Virology, University Medical Center Rotterdam,

Dr. Molewaterplein 50,

3015 GE Rotterdam, The Netherlands

D. M. Burger

Department of Clinical Pharmacy, Radboud University Nijmegen

Medical Centre,

Geert Grooteplein 10,

6525 GA Nijmegen, The Netherlands

N. G. Hartwig

Department of Paediatrics, Division Infectious

Diseases/Immunology, University Medical Center

Rotterdam-Sophia Children's Hospital,

Dr. Molewaterplein 60,

3015 GJ Rotterdam, The Netherlands trations were determined by matrix-assisted laser desorption/ionization-triple quadrupole tandem mass spectrometry (MALDI-QqQ-MS/MS). The application of DBS made it possible to measure lopinavir and ritonavir in whole blood in therapeutically relevant concentrations. The MALDI-QqQ-MS/MS plasma assay was successfully cross-validated with a commonly used high-performance liquid chromatography (HPLC)-ultraviolet (UV) assay for the therapeutic drug monitoring (TDM) of HIV-1-infected patients, and it showed comparable performance characteristics. Observed DBS concentrations showed as well, a good correlation between plasma concentrations obtained by MALDI-QqQ-MS/MS and those obtained by the HPLCUV assay. Application of DBS for TDM proved to be a good alternative to the normally used plasma screening. Moreover, collection of DBS requires small amounts of whole blood which can be easily performed especially in (very) young children where collection of large whole blood amounts is often not possible. DBS is perfectly suited for TDM of HIV-1-infected children; but nevertheless, DBS can also easily be applied for TDM of patients in areas with limited or no laboratory facilities.

Keywords Protease inhibitors - Dried blood spots . MALDI-QqQ-MS/MS · HIV-1 · Lopinavir · Ritonavir

\section{Introduction}

Protease inhibitors (PI) belong to a class of pharmaceuticals used in the treatment of the human immunodeficiency virus infection (HIV) [1]. This class of protease inhibitor drugs inhibits the viral HIV-1 protease enzyme, which processes viral polyproteins which are essential for the completion of the HIV viral life cycle. PI drugs are used within the 
combined antiretroviral therapy, a therapy which has significantly improved the decrease in morbidity and mortality for HIV-infected patients [2]. Kaletra ${ }^{\circledR}$ (Abbott) is a co-formulation, containing the two protease inhibitors lopinavir (LPV, $80 \mathrm{mg} / \mathrm{mL}$ ) and ritonavir (RTV, $20 \mathrm{mg} / \mathrm{mL}$ ). This co-formulation is used in pediatric HIV-1 pediatric treatment. RTV acts to boost up the lopinavir plasma concentration by inhibition of the cytochrome P450 CYP3A isozymes [3] and the active transport by the P-glycoprotein [4]. Patient's drug intake compliance is very important in HIV treatment, and the patient's compliance with the drug regimen can be monitored by determination of the LPV and RTV plasma concentration levels. More importantly, plasma concentrations of HIV protease inhibitors are used to optimize dosing with these drugs for each individual patient (therapeutic drug monitoring (TDM)), which is in particular important for HIV-infected children because of the profound changes in drug pharmacokinetics during development and maturation of the child.

Patient compliance to the HIV drugs regimen and TDM is in general monitored by analyses of plasma or serum; sometimes, also another body fluid such as saliva [5] is used. Heine et al. [6] and Koal et al. [7] used both DBS for the quantitative determination of PI concentrations by LC-MS/MS. The DBS assay was first described by Guthrie [8] in the 1960s and was primarily used to screen neonates for metabolic diseases. DBS assays are not only applied for screening of metabolic diseases in neonates but in general can also be used to determine drug concentrations in whole blood. Especially, DBS assays have been applied for the determination of concentrations of antimalarials [9], antiepileptics [10], and antiretrovirals [7].

Whole blood is collected from either heel or finger prick or by collection by vena-puncture. The whole blood is then blotted onto a DBS collection card. After the whole blood spots have been dried, a disk is punched out from the center of the DBS, and PI are extracted from the disk with appropriate solvents and extracts are analyzed. Over the last decades, several DBS assays have been applied for screening of metabolic disorders [11].

Analytical assays used for the determination of LPV, RTV, and other PI in general are high-performance liquid chromatography (HPLC) with ultraviolet (UV) [12] detection, electrospray ionization-mass spectrometry (ESI-MS) [13, 14] and tandem mass spectrometry (ESI-MS/MS) [7, 15-18], and matrix-assisted laser desorption/ionization-triple quadrupole tandem mass spectrometry (MALDI-QqQ-MS/MS) [19]. MALDI-QqQ-MS/ MS is a relative new technique that combines two known aspects of mass spectrometry, MALDI and triple quadrupole mass spectrometry $(\mathrm{QqQ})$, into a novel merged mass spectrometric technique. Biological samples are mixed with a solution containing the MALDI matrix, which is typically a small molecule that absorbs the energy of the laser and assists with desorption and gentle ionization of the analyte. A small aliquot $(0.25-1.5 \mu \mathrm{L})$ of the sample/ matrix solution is then spotted onto a MALDI (stainless steel) target plate. The spots are dried at ambient temperature and analyzed by ionization by a high repetition rate solid state UV laser $(1 \mathrm{kHz}, 349 \mathrm{~nm})$. The produced ions are then analyzed using multiple reaction monitoring (MRM). The MALDI-QqQ-MS/MS technique does not necessarily require liquid chromatographic separation of samples prior to mass MRM analyses. Therefore, an analysis time of MALDI-QqQ-MS/MS is primarily determined by the amount of laser shots required to obtain a reproducible MRM, the raster speed of the laser, and the repetition rate of the laser. Using our settings, the analysis takes $5 \mathrm{~s}$ per sample or even less (depending on laser speed). The MALDI-QqQ-MS/MS assay has been previously used to determine the concentrations of different drug types [20], antiretroviral drugs [21, 22], benzodiazepines [23], beta-blockers, and antibiotics [24] but was also successfully used as a screening tool in enzyme kinetic studies [25]. In this study, we present two newly developed MALDI-QqQ-MS/MS assays for ultrafast and high-throughput monitoring of lopinavir and ritonavir drug concentrations in plasma and in whole blood (DBS) from HIV-1-infected children. The new assays were validated according to the FDA guidelines [26], and tested and applied for its clinical use by analyses of DBS and plasma samples from a cohort study of HIV-1-infected children. Observed plasma concentration levels of LPV and RTV by the MALDI-QqQ-MS/MS plasma assay were also cross-validated with a validated HPLC-UV assay.

\section{Materials and methods}

\section{Chemicals and reagents}

Investigated PI drugs were a kindly donation from pharmaceutical companies: LPV and RTV by Abbott Laboratories (IL, USA) and the internal standard nelfinavir (NFV) by Pfizer (Groton, CT, USA). A primary stock solution of LPV and RTV was prepared in methanol and contained $200 \mu \mathrm{mol} / \mathrm{L}$ LPV and RTV, while a second primary stock solution also prepared in methanol contained $200 \mu \mathrm{mol} / \mathrm{L}$ of the internal standard NFV, respectively. All solvents used were obtained from commercial sources and were of LC-MS grade (Biosolve, Valkenswaard, the Netherlands), and other chemicals were of ACS grade from Sigma Aldrich (Zwijndrecht, the Netherlands). Chemical 


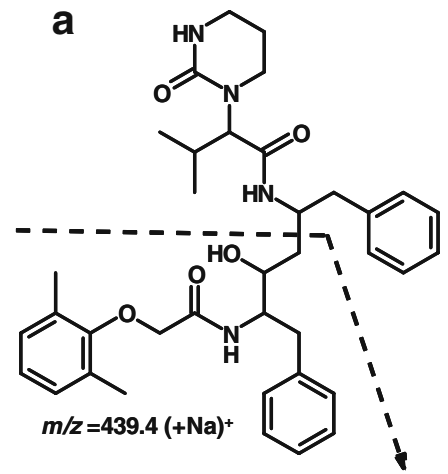

$m / z 651.4>m / z 439.4$

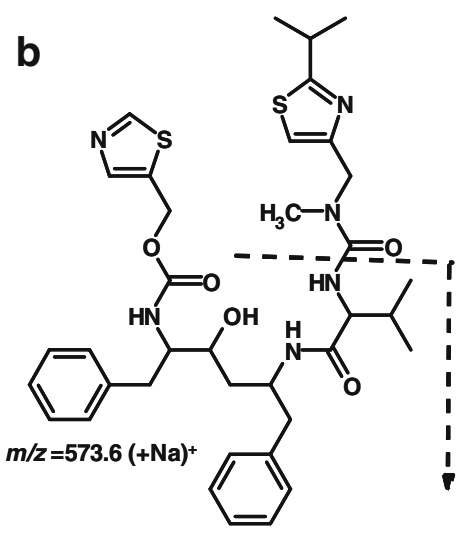

$m / z 743.4>m / z 573.6$

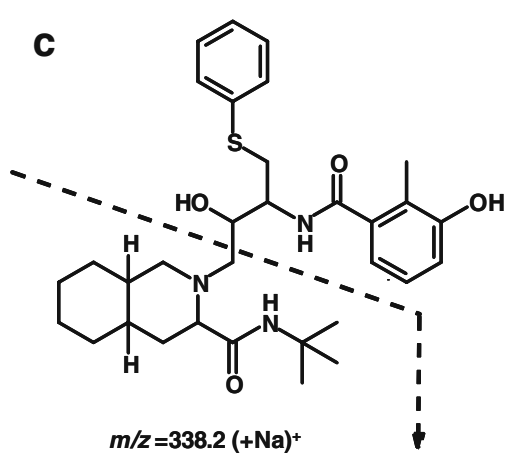

$m / z 590.0>m / z 338.2$

Fig. 1 Molecular structures of a LPV, b RTV, and $\mathbf{c}$ internal standard NFV and respective fragmentations of the sodium adducts of LPV, RTV, and NFV and MRM transition

structures of lopinavir, ritonavir, and used internal standard nelfinavir are illustrated in Fig. 1.

Preparation of calibrators and calibration curves

\section{Whole blood-based calibrators for DBS analyses}

Calibrators for the DBS assay were prepared by dilution of the primary stock solution with drug-free whole blood (EDTA) from healthy donors that were obtained from the local blood bank (Sanquin Blood Supply Foundation, Rotterdam, the Netherlands). Whole blood-based calibrators yielded following concentrations: 50, 25, 10, 5.0, $2.5,1.0,0.5$, and $0.25 \mu \mathrm{mol} / \mathrm{L} \mathrm{LPV}$ and RTV. From the calibrators, an aliquot of $25 \mu \mathrm{L}$ were spotted $(n=4)$ onto FTA PK DBS collecting cards from GE Healthcare (Piscataway, NJ, USA). DBS collecting cards were dried overnight at room temperature. Two disks (7 $\mathrm{mm}$ diameter) were punched out from two different DBS, and punches were transferred into a safe-lock micro tube (1.5 mL, Eppendorf) and extracted with $250 \mu \mathrm{L}$ of a acetonitrile/water mixture 80:20 ( $v / v)$ containing $5 \mu \mathrm{mol} / \mathrm{L}$ nelfinavir as internal standard (IS) for $30 \mathrm{~min}$ in an ultrasonic bath. DBS extracts were centrifuged for $5 \mathrm{~min}$ at room temperature $(2,000 \times g)$ to remove precipitated proteins. From the supernatants, $20 \mu \mathrm{L}$ was mixed with $20 \mu \mathrm{L}$ of MALDI matrix $\alpha$-cyano-4-hydroxycinnamic acid ( $\alpha$-CHCA), Agilent Technologies; $6.2 \mathrm{mg} / \mathrm{mL}$ in methanol/acetonitrile/water 36:56:8 $(v / v / v), \mathrm{pH}=2.5$ supplemented with $1 \mathrm{mmol} / \mathrm{L}$ sodium iodide $(\mathrm{NaI})$ and $1.5 \mu \mathrm{L}$ of the supernatant/MALDI matrix mixtures were spotted $(n=5)$ on an Opti-TOF 96-well stainless steel target plate $(123 \times 81 \mathrm{~mm}$; MDS Analytical Technologies, Concord, Canada), and sample spots were dried at room temperature for $5 \mathrm{~min}$.

\section{Plasma-based calibrators}

Plasma-based calibrators were prepared from drug-free plasma harvested from the drug-free whole blood collected of healthy donors, which were also used for development of the DBS assay. The plasma was prepared by centrifugation of whole blood for $10 \mathrm{~min}$ at a temperature of $4{ }^{\circ} \mathrm{C}$ $(400 \times g)$. Plasma-based calibrators were prepared by spiking with LPV and RTV and yielded the following calibrators: 4, $1,0.5,0.2,0.1$, and $0.05 \mu \mathrm{mol} / \mathrm{L}$, and a blank. From each calibrator, two aliquots of $10 \mu \mathrm{L}$ were spiked with $5 \mu \mathrm{L}$ of an IS solution and were deproteinized by the addition of $85 \mu \mathrm{L}$ of acetone. Precipitated proteins were removed by centrifugation for $5 \mathrm{~min}$ at room temperature $(2,000 \times \mathrm{g})$. The supernatants $(20 \mu \mathrm{L})$ were mixed with $\alpha$-CHCA solution $(20 \mu \mathrm{L}), 1.5 \mu \mathrm{L}$ of the supernatant/matrix mixture was spotted $(n=5)$, and the resulting sample spots were dried at room temperature for $5 \mathrm{~min}$.

\section{MALDI-triple quadrupole tandem mass spectrometry}

MALDI-QqQ-MS/MS analyses were conducted on a Flashquant Workstation combined with a 4000 API mass analyzer (MDS Analytical Technologies) operating in the positive ionization mode. MS/MS analyses were carried out by MRM at unit resolution. MRM parameters of the sodium adducts of analyte molecules $(\mathrm{M}+\mathrm{Na})^{+}$used in this study were LPV $(\mathrm{m} / \mathrm{z}$ 651.4>439.4), RTV $(\mathrm{m} / \mathrm{z}$ 743.4>573.6), and NFV $(\mathrm{m} / \mathrm{z}$ 590.0>338.2) and collision energies were 52, 50, and $50 \mathrm{~V}$ for LPV, RTV, and NFV, respectively. Optimized MALDI-QqQ-MS/MS instrument parameters were as follows: laser power $60 \%$, target plate voltage $60 \mathrm{~V}$, skimmer voltage $0 \mathrm{~V}, \mathrm{CAD}$ gas 6 arbitrary units (collision gas), collision cell energy exit potential (CXP) $10 \mathrm{~V}$, source gas 10 arbitrary units, dwell time $20 \mathrm{~ms}$, and laser speed $1 \mathrm{~mm} / \mathrm{s}$. 
MALDI-MS instrument control and data analyses were performed using Flashquant 1.0 software and Analyst 1.4.2 application software (MDS Analytical Technologies).

\section{Assay validation}

The newly developed assays were validated for the following assay specific properties; limit of detection (LOD), lower limit of quantification (LLOQ), linearity, accuracy, precision, and stability of LPV and RTV in DBS/ plasma matrix using the FDA's Guidance for Industry on bio-analytical method validation procedure [26]. Assay precision, accuracy, and the stability of the analytes were determined from quality control samples (QC) prepared in drug-free whole blood and drug-free plasma by spiking LPV and RTV at three different concentrations of 50, 10, and $1.0 \mu \mathrm{mol} / \mathrm{L}$ for DBS analysis and 4.0, 2.0, and $1.0 \mu \mathrm{mol} / \mathrm{L}$ for plasma analysis, respectively.

\section{Children cohort study and sample preparation}

Nineteen HIV-1-infected children $(n=19)$ were included into the cohort patient study during a 2-month period. Average age of the participants was $10.2 \pm 4.0$ years. Whole blood samples from participants were collected at the outpatient clinic of the Pediatrics department at the Erasmus MC-Sophia Children Hospital (Rotterdam, the Netherlands). Patient blood samples were collected in compliance with the Helsinki. The whole blood samples from 16 of the 19 participants were collected at approximately the same time after oral intake of Kaletra $^{\circledR}$ (mean collection time of $15.6 \pm 0.7 \mathrm{~h}$ ). From the three remaining patients, whole blood samples were collected at $4.0,18.25$, and $20.50 \mathrm{~h}$, respectively. Whole blood samples were drawn by vena-puncture and collected into BD Vacutainer EDTA tubes (Becton Dickinson, Breda, the Netherlands). For the DBS assay, $4 \times 25 \mu \mathrm{L}$ of collected whole blood was blotted onto a DBS collection card followed by overnight drying of the DBS collection card at ambient temperature. After the DBS collection cards were dried, they were stored in a plastic-sealable bag (Minigrip, Lelystad, the Netherlands) at a temperature of $4{ }^{\circ} \mathrm{C}$. Plasma was prepared from an aliquot of collected EDTA whole blood by centrifugation for $6 \min \left(400 \times g, 4^{\circ} \mathrm{C}\right)$. Plasma was separated from the red blood cell pellet transferred into a separate vial and directly stored in a freezer at a temperature of $-80^{\circ} \mathrm{C}$.

\section{Results}

Assay development

The DBS assay was developed by spiking whole blood collected from healthy donors with LPV, RTV, and internal standard NFV. First, extraction of the PI from the DBS matrix was optimized by blotting aliquots of $25 \mu \mathrm{L}$ of spiked whole blood $(20 \mu \mathrm{mol} / \mathrm{L}$ LPV and RTV) on DBS collection cards. After drying, the DBS were punched out and extracted with different organic solvent/water mixtures. Combinations of methanol/water $\left(\mathrm{MeOH} / \mathrm{H}_{2} \mathrm{O}\right)$ mixtures ranged from $\mathrm{MeOH} / \mathrm{H}_{2} \mathrm{O}$ 10:90 (v/v) to $\mathrm{MeOH} / \mathrm{H}_{2} \mathrm{O}$ 90:10 $(v / v)$, and the same procedure was used for acetonitrile/ water $\left(\mathrm{ACN} / \mathrm{H}_{2} \mathrm{O}\right)$ solvent mixtures to determine optimal conditions for PI measurement in organic/water solutions. The best extraction results for LPV, RTV, and NFV were obtained with $\mathrm{ACN} / \mathrm{H}_{2} \mathrm{O}$ 80:20 $(v / v)$ solvent mixtures. It was observed that extracts with higher water content $(>20 \% v / v)$ were colored slightly to deep red/brown. These extracts showed severe ion suppression during MALDI ionization, which is probably due to extracted (oxidized) hemoglobin. Moreover, more profound ion suppression was observed with $\mathrm{MeOH} / \mathrm{H}_{2} \mathrm{O}$ mixtures and less with $\mathrm{ACN} /$ $\mathrm{H}_{2} \mathrm{O}$ mixtures. Ion suppression effects decreased significantly for $\mathrm{ACN} / \mathrm{H}_{2} \mathrm{O}$ with higher ACN content $(>40 \% v / v)$. Subsequently, we studied the maximum amount of whole blood that could be blotted onto the DBS collection cards without overloading the spot and causing it to spread. Volumes of $5,15,25$, and $40 \mu \mathrm{L}$ of spiked whole blood were blotted onto DBS collection cards. The $40 \mu \mathrm{L}$ volume resulted in a spot that had a larger diameter than the maximum allowed by the DBS (marked by a printed circle); thus, adjacent spots ran into each other. The optimal whole blood amount that could be blotted was $25 \mu \mathrm{L}$, covering approx. $90 \%$ of the allowed surface of printed circles. Smaller whole blood amounts were also tested, but these resulted in significantly higher detection limits. After determination of the optimal amount of whole blood, extraction volume and extraction time of DBS were studied by the application of $50,100,250$, and $500 \mu \mathrm{L}$ of $\mathrm{ACN} /$ $\mathrm{H}_{2} \mathrm{O} 80: 20(v / v)$ solvent mixture and extraction times of 15, 30,45 , and 60 min using sonication. The optimal DBS extraction volume and extraction time were observed for $250 \mu \mathrm{L}$ of $\mathrm{ACN} / \mathrm{H}_{2} \mathrm{O}$ 80:20 $(v / v)$ mixture and extraction time of $30 \mathrm{~min}$.

The plasma assay was developed by the application of spiked plasma prepared from the drug-free whole blood that was also used for the DBS assay development. To this end, plasma was spiked with LPV, RTV, and NFV at a concentration level of $1 \mu \mathrm{mol} / \mathrm{L}$. Deproteinization of plasma was studied by the application of different deproteinization agents. Studied deproteinization agents were ACN, acetone (ACT), and $\mathrm{MeOH}$. Aliquots of $10 \mu \mathrm{L}$ of spiked plasma were deproteinized by the addition of $90 \mu \mathrm{L}$ of $\mathrm{ACN}, \mathrm{ACT}$, or $\mathrm{MeOH}$ and mixed on a vortex mixer for $1 \mathrm{~min}$. Precipitated proteins were removed by centrifugation for $10 \mathrm{~min}(2,000 \times \mathrm{g})$ at room temperature, and supernatants were mixed with MALDI matrix and spotted. The highest recoveries were observed for 
ACT deproteinization and were $93.4 \%, 93.2 \%$, and $98.5 \%$ for LPV, RTV, and NFV, respectively. Recovery rates for LPV, RTV, and NFV using acetonitrile and methanol were approx. $80 \%$ and $13 \%$, respectively. Furthermore, the amount of ACT used for deproteinization was optimized, and following amounts of ACT were tested on their deproteinization capacities; $40,60,80,90$, and $100 \mu \mathrm{L}$ for the deproteinization of $10 \mu \mathrm{L}$ plasma. Observed recovery rates for LPV were $36.8 \%, 59.7 \%, 114.0 \%, 93.8 \%$, and $85.4 \%$; for RTV $29.6 \%$, $64.6 \%, 111.3 \%, 92.3 \%$, and $77.6 \%$; and for NFV $28.4 \%$, $69.9 \%, 108.5 \%, 98.5 \%$, and $86.7 \%$, respectively. Further deproteinization of plasma samples was performed by deproteinization of $10 \mu \mathrm{L}$ of plasma sample with $90 \mu \mathrm{L}$ of acetone.

Assay validation

\section{Linearity of DBS and plasma assays}

Selected range of whole blood and plasma-based calibrators indicated the existence of linear relationships between the ratio of the peak areas of LPV/NFV ratio and RTV/NFV ratio (y) and concentration $(x)$ of LPV and RTV in DBS and plasma, respectively. All calibration curves showed linear relationships between the analyte peak areas and analyte concentration. For the plasma assay, the calibration curves were linear between 0.05 and $4.0 \mu \mathrm{mol} / \mathrm{L}$ and between 0.25 and $50 \mu \mathrm{mol} / \mathrm{L}$ for the DBS assay, respectively. The linear regression analyses were calculated using GraphPad Prism software version 5.00 for windows (GraphPad Software, San Diego, CA, USA). The regression equations of the DBS assay were $y=0.022 x-0.0069(\mathrm{LPV})$ and $y=0.0165 x-$ 0.0096 (RTV), and regression coefficients were 0.9941 and 0.9948 for LPV and RTV, respectively. The calibration curve regression equation and regression coefficients of the plasma assay were $y=4.5131 x-0.0208$ (LPV) and $y=30.0478 x-$ 0.1904 (RTV), and regression coefficients were 0.9932 and 0.9965 for LPV and RTV, respectively.

\section{LLOQ and LOD of DBS and plasma assay}

The LLOQ was defined as the lowest matrix-based calibrator of the calibration curve that could be measured with acceptable accuracy and precision $(<20 \%$ error $)$ in compliance with FDA regulations. The LLOQ of the DBS assay was $0.25 \mu \mathrm{mol} / \mathrm{L}$ for $\mathrm{LPV}$ as well as RTV and $0.05 \mu \mathrm{mol} / \mathrm{L}$ for both analytes for the plasma assay. The upper limits of quantification was defined as the highest matrix-based calibrator measured and was $200 \mu \mathrm{mol} / \mathrm{L}$ for LPV and RTV for the DBS assay and $4.0 \mu \mathrm{mol} / \mathrm{L}$ for the plasma assay, respectively. The LODs, defined as three times the signal-to-noise ratio of drug-free whole blood/ plasma, were $0.039 \mu \mathrm{mol} / \mathrm{L}(\mathrm{LPV})$ and $0.058 \mu \mathrm{mol} / \mathrm{L}$
(RTV) for the DBS assay, and $0.0017 \mu \mathrm{mol} / \mathrm{L}$ (LPV) and $0.0093 \mu \mathrm{mol} / \mathrm{L}$ (RTV) for the plasma assay, respectively.

\section{Within- and between-run accuracy and precision}

The accuracy and precision of the DBS and plasma assay for LPV and RTV were in good compliance with the FDA regulations $(<15 / 20 \%$ error and $\% \mathrm{CV})$. Observed accuracy and precision results at three studied concentration levels are listed in Table 1.

\section{Stability of LPV and RTV}

LPV and RTV were considered stable in the DBS and plasma matrix if during conservation of the quality control samples the decrease in concentration was $<15 \%$ (expressed as \% error). The observed stability of LPV and RTV in both biological matrices was $<15 \%$ error for studied concentration levels for storage in a refrigerator $\left(24 \mathrm{~h}, 4^{\circ} \mathrm{C}\right), 20$ days in a freezer $\left(-20^{\circ} \mathrm{C}\right)$, and during three freeze-thaw cycles (Tables 2 and 3).

\section{Cross-validation of plasma assay with HPLC-UV assay}

Plasma LPV and RTV concentrations observed by the newly developed MALDI-QqQ-MS/MS plasma assay were crossvalidated with an HPLC-UV method as described previously [27]. HPLC-UV assay determinations of plasma LPV and RTV concentrations of participants from the cohort study were performed at the Dept. Clinical Pharmacy (Radboud University Nijmegen Medical Centre, the Netherlands). For cross-validation purposes, plasma samples were separately prepared for analysis at both laboratories, and standard solutions were not exchanged between the laboratories.

\section{Discussion}

Currently, several papers describe the determination of PI concentrations and other antiretroviral drugs in plasma from HIV-1-infected patients. All papers applied HPLC or LC assays in combination with UV or mass spectrometric detection for the determination of drug concentrations. Moreover, the average analysis time of applied assays ranged between $5 \mathrm{~min}$ to approx. $20 \mathrm{~min}$ per sample depending on instrumentation and separation column used. Previously, we demonstrated that the measurement of intracellular concentrations of LPV and RTV in lysates of peripheral blood mononuclear cells (PBMC) was not only very sensitive (femtomol amounts) but as ultrafast and high-throughput [19] compared with the LCMS/MS assay published by Pelerine et al. [12] where an analysis time of $20 \mathrm{~min}$ was reported. The MALDI-QqQ-MS/ MS assay analysis time for one sample was $<15 \mathrm{~s}$, which 
Table 1 Precision and accuracy of the MALDI-QqQ-MS/MS assay at three DBS and plasma concentration levels

\begin{tabular}{|c|c|c|c|c|c|c|c|c|c|c|c|c|}
\hline \multirow[t]{4}{*}{ Analyte } & \multicolumn{6}{|c|}{ Dried blood spots (DBS) } & \multicolumn{6}{|c|}{ Plasma } \\
\hline & \multicolumn{12}{|c|}{ Nominal concentration $(\mu \mathrm{mol} / \mathrm{L})$} \\
\hline & \multicolumn{2}{|l|}{50.0} & \multicolumn{2}{|l|}{10.0} & \multicolumn{2}{|l|}{1.0} & \multicolumn{2}{|l|}{4.0} & \multicolumn{2}{|l|}{2.0} & \multicolumn{2}{|l|}{1.0} \\
\hline & LPV & RTV & LPV & RTV & LPV & RTV & LPV & RTV & LPV & RTV & LPV & RTV \\
\hline \multicolumn{13}{|l|}{ Within-run validation $^{\mathrm{a}}$} \\
\hline Mean observed concentration $(\mu \mathrm{mol} / \mathrm{L})$ & 49.9 & 50.7 & 10.7 & 10.6 & 0.9 & 0.9 & 3.7 & 4.0 & 1.9 & 2.1 & 1.1 & 1.2 \\
\hline Accuracy $\left(\%\right.$ error $\left.^{b}\right)$ & -0.3 & 1.4 & 7.2 & 5.6 & -8.1 & -10.8 & -8.6 & 0.5 & -3.7 & 5.2 & 8.8 & 12.4 \\
\hline Precision $(\% \mathrm{CV})$ & 4.7 & 6.4 & 11.7 & 11.9 & 16.5 & 12.8 & 7.5 & 12.5 & 10.3 & 13.4 & 11.2 & 8.8 \\
\hline \multicolumn{13}{|l|}{ Between-run validation $^{\mathrm{c}}$} \\
\hline Mean observed concentration $(\mu \mathrm{mol} / \mathrm{L})$ & 51.2 & 49.4 & 10.9 & 9.6 & 1.2 & 1.0 & 3.7 & 4.2 & 2.0 & 2.2 & 1.1 & 1.1 \\
\hline Accuracy $\left(\%\right.$ error $\left.^{\mathrm{b}}\right)$ & 2.4 & -1.2 & 9.0 & -4.3 & 10.1 & 1.0 & -7.1 & 3.8 & 1.5 & 10.8 & 8.4 & 13.7 \\
\hline Precision $(\% \mathrm{CV})$ & 11.4 & 9.8 & 12.2 & 11.3 & 18.2 & 18.3 & 12.0 & 14.6 & 13.2 & 12.4 & 12.1 & 11.0 \\
\hline
\end{tabular}

${ }^{\mathrm{a}}$ Within-run results summarize ten spots per QC sample at each concentration level in one experiment

$\mathrm{b}_{\%}$ error $=($ mean observed concentration - nominal concentration $) /($ nominal concentration $) \times 100 \%$

${ }^{\mathrm{c}}$ Between-run results summarize three different experiments from three consecutive days with ten spots per QC sample at each concentration level

means that a full 96-well MALDI target plate could be analyzed within 16- to 17-min analysis time.

For the measurement of LPV and RTV concentrations in plasma and DBS, two new bio-analytical assays were developed and validated. In the past, we already applied the MALDI-QqQ-MS/MS for the analysis of LPV and RTV in plasma samples, but this method was not validated in compliance with the FDA regulations [19]. A crossvalidation of the DBS assay was not possible because no HPLC-UV assay applying DBS samples was available, but for the validation of the plasma assay, an accredited HPLC-UV was used. The DBS and plasma assays were developed using quality control samples, and both assays were validated in full compliance with the FDA regulations [26]. NFV was selected as internal standard because it was in the past successfully used in the assay for the intracellular PBMC concentrations for LPV and RTV [19]. Observed LOD of the DBS assay for LPV and RTV were 0.039 and $0.058 \mu \mathrm{mol} / \mathrm{L}$, respectively. Observed LODs were higher (2.8 times (LPV) and 3.6 times higher (RTV)) than reported LODs by Koal and coworkers [7]. In a reported work by Koal et al. [7], they injected $3.125 \mu \mathrm{L}$ whole blood (calculated from extracted amount whole blood) from extracted $5 \mu \mathrm{L}$ whole blood into the LC-MS instrumentation; this in contrast to the MALDI-QqQ-MS/ MS assay where $0.05 \mu \mathrm{L}$ of the $25 \mu \mathrm{L}$ DBS was used for

Table 2 Stability experiments with DBS-QC samples containing LPV and RTV under different storage conditions

\begin{tabular}{|c|c|c|c|c|c|c|c|c|}
\hline \multirow[t]{5}{*}{ Nominal concentration $(\mu \mathrm{mol} / \mathrm{L})$} & \multicolumn{8}{|c|}{ Storage conditions/time } \\
\hline & \multicolumn{4}{|c|}{ Refrigerator $\left(4^{\circ} \mathrm{C}\right)^{\mathrm{a}}$} & \multicolumn{4}{|c|}{ Dessicator $\left(20^{\circ} \mathrm{C}\right)^{\mathrm{a}}$} \\
\hline & \multicolumn{4}{|l|}{$24 \mathrm{~h}$} & \multicolumn{4}{|l|}{ 20days } \\
\hline & \multicolumn{2}{|l|}{ LPV } & \multicolumn{2}{|l|}{ RTV } & \multicolumn{2}{|l|}{ LPV } & \multicolumn{2}{|l|}{ RTV } \\
\hline & Mean (SD) & $\%$ error $^{\mathrm{b}}$ & Mean (SD) & $\%$ error $^{\mathrm{b}}$ & Mean (SD) & $\%$ error $^{\mathrm{b}}$ & Mean (SD) & $\%$ error $^{\mathrm{b}}$ \\
\hline 50.0 & $49.9(6.1)$ & -0.2 & $48.5(4.0)$ & -3.0 & $49.7(3.3)$ & -0.6 & $49.2(3.9)$ & -1.6 \\
\hline 10.0 & $10.5(0.9)$ & 5.0 & $10.4(1.4)$ & 4.0 & $9.4(1.1)$ & -6.0 & $10.1(0.7)$ & 1.0 \\
\hline 1.0 & $1.1(0.1)$ & 10.0 & $0.9(0.1)$ & -10.0 & $0.93(0.15)$ & -7.0 & $1.1(0.2)$ & 10.0 \\
\hline
\end{tabular}

${ }^{\mathrm{a}}$ Results summarize ten spots per QC sample at each concentration level in one experiment

$\mathrm{b}_{\%}$ error $=($ mean observed concentration - nominal concentration $) /($ nominal concentration $) \times 100 \%$ 
analysis. Moreover, the LODs of the MALDI-QqQ-MS/MS plasma assay were 0.0017 and $0.0093 \mu \mathrm{mol} / \mathrm{L}$ for LPV and RTV, respectively, comparable with LODs published by Koal et al. [7], but they applied a total sample amount of $1.67 \mu \mathrm{L}$ of plasma for the LC-MS/MS analysis and the MALDI-QqQ-MS/MS assay just $0.05 \mu \mathrm{L}$.

By calculating the total injected sample amounts of $0.05 \mu \mathrm{L}$ of sample for DBS as well as plasma assay compared with the amounts used in the reported LC-MS/ MS assay [7], it could be concluded that both MALDI-QqQ-MS/MS assays were approx. 30 times more sensitive. Besides the higher sensitivity, less sample amount is needed. The more significant advantages of the application of the MALDI-QqQ-MS/MS technology are the ultrafast and high-throughput characteristics of this technology. Although LODs of the MALDI-QqQ-MS/MS assays are higher than, for example, LC-MS/MS, MALDI-QqQ-MS/MS is sensitive enough to be applied in the clinical practice. In very short time, TDM of DBS and plasma concentrations from HIV-1-infected children could be applied to verify if clinical relevant systemic concentrations were present. Almost all patients had therapeutically relevant concentrations of LPV in collected plasma and via DBS collected whole blood samples. Expected therapeutically relevant concentrations had to range between 12 and $20 \mu \mathrm{mol} / \mathrm{L}$. This relative broad range relates if the HIV- 1 child was native to Kaletra ${ }^{\circledR}$ or already previously treated with Kaletra ${ }^{\circledR}$ [28].

The assays described here were validated by the determination of linearity, recovery rates, within- and between-run accuracy, and precision and stability of LPV and RTV in both DBS and plasma matrix. The within- and between-run accuracy and precision as well as stability were all in compliance with FDA regulation for validation of (new) bio-analytical assays (Tables 1, 2, and 3). After full validation of both assays, real patient samples from a cohort study were measured.

Patients DBS samples were extracted and analyzed by the MALDI-QqQ-MS/MS DBS assay, and corresponding plasma samples were prepared and analyzed in duplicate. Patients plasma samples $(n=19)$ were prepared according to the optimal conditions determined. Samples $(1 \mu \mathrm{L})$ were spotted in fivefold onto the MALDI target plate. Patient's DBS were punched out in duplicate, extracted, and spotted in fivefold onto the MALDI target plate. Comparison of observed plasma concentrations and DBS concentrations of LPV and RTV showed a significant correlation. The correlation between patient's plasma concentrations and DBS concentrations of LPV and RTV are illustrated in Fig. 2.

Observed plasma concentrations of LPV and RTV by the MALDI-QqQ-MS/MS plasma assay were also cross- 
Fig. 2 Comparison of drug levels in plasma and DBS samples from HIV-1-infected children: a LPV and b RTV
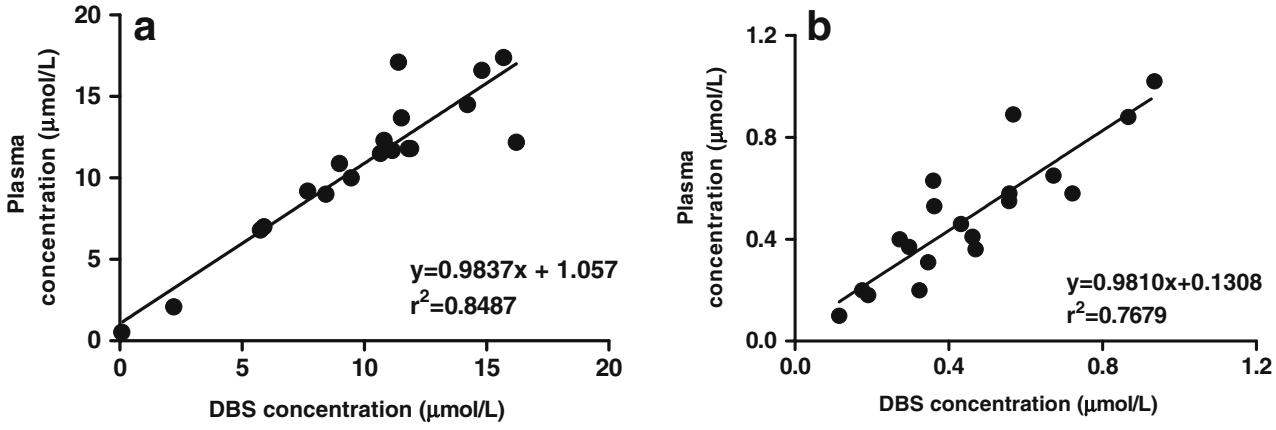

validated with measured plasma concentrations by a validated HPLC-UV [27] at an accredited laboratory of the Dept. of Clinical Pharmacy (Radboud University Nijmegen Medical Centre, the Netherlands) by calculating the bias between both methods according to Bland-Altman method comparison statistics [29, 30]. The differences for LPV and RTV concentrations observed by both assays are expressed as a Bland-Altman plot and are illustrated in Fig. 3, and the linear regression between observed LPV and RTV concentrations patient's plasma samples of the HPLCUV- and MALDI-QqQ-MS/MS assay are also presented. All measured differences in concentrations for LPV and RTV for both analytical assays were between the mean of both assays $\pm 2 \mathrm{SD}$ (95\% confidence), except for one patient's sample (RTV) (Fig. 3d).
Both LPV and RTV concentrations were statistically compared; however, from clinical point of view, the protease inhibitor LPV is more important than RTV because LPV is the active drug responsible for inhibition of the viral HIV-1 protease. RTV, as used in the concentration in the co-formulated Kaletra medication, acts as a kind of pharmacokinetic booster of LPV, preventing the metabolic degradation by drug-metabolizing CYP3A isozymes. In the clinical practice, the LPV concentration is important for TDM and for determination of the patient's compliance with the drug regimen. The MALDI-QqQ-MS/MS plasma assay shows a slightly positive bias compared with the HPLC-UV assay (Fig. 3b), while almost no bias is observed with RTV (Fig. 3d) analysis. For the observed positive bias of the LPV determination, no explanation could be given.
Fig. 3 a Comparison of observed LPV concentrations by HPLC-UV assay and newly developed MALDI-QqQ-MS/ MS assay. b Bland-Altman plot of differences between observed LPV concentrations by MALDI-QqQ-MS/MS and HPLC-UV assay. c Comparison of observed RTV concentrations by HPLC-UV assay and the new MALDI-QqQ-MS/MS assay. d Bland-Altman plot of differences between observed RTV concentrations by

MALDI-QqQ-MS/MS and HPLC-UV assay. Patients $(n=19)$ LPV and RTV plasma concentrations were measured by the newly developed MALDI-QqQ-MS/MS plasma assay and cross-validated with an accredited HPLC-UV assay. Solid line represents mean, and dotted lines represent mean \pm 2 SD (standard deviation of mean)
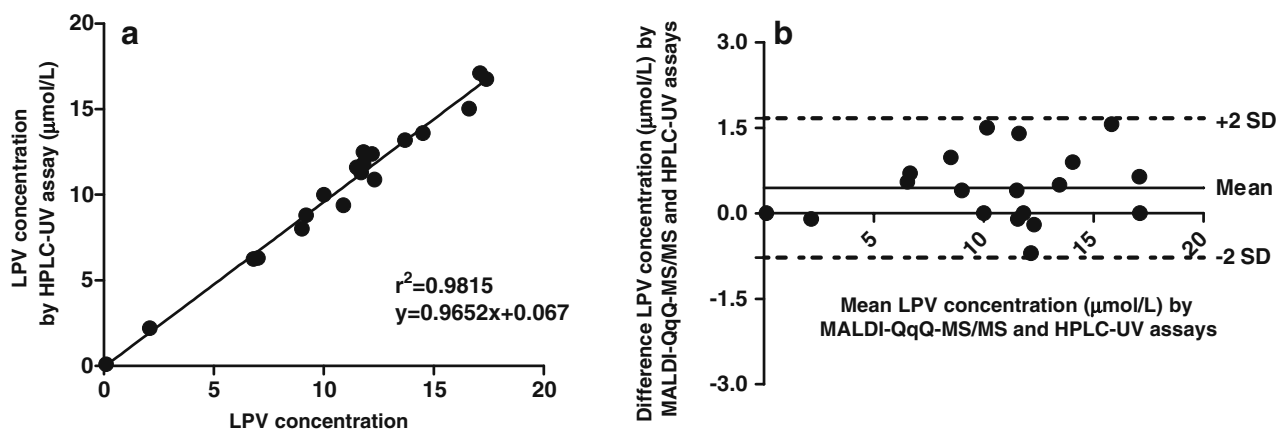

by MALDI-QqQ-MS/MS assay ( $\mu \mathrm{mol} / \mathrm{L}$ )
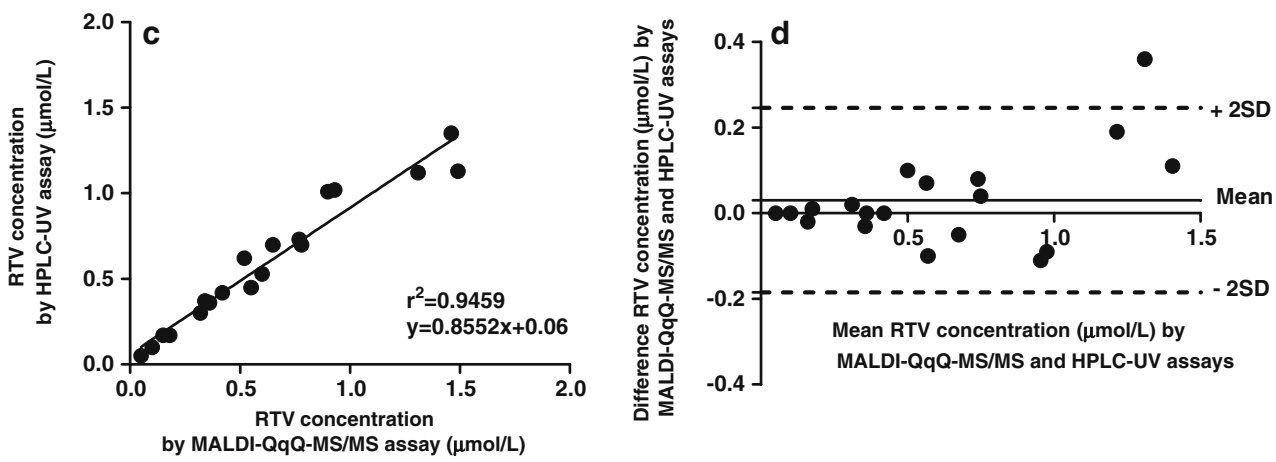
In conclusion, the newly developed MALDI-QqQ-MS/MS assays described here can be used for the sensitive and ultrafast determination of the antiretroviral drugs LPV and RTV in plasma and in DBS samples from HIV-1-infected children. The assays showed significant correlations between observed LPV and RTV concentrations in whole blood (DBS) and in plasma. The MALDI-QqQ-MS/MS plasma assay was successfully cross-validated with an accredited HPLC-UV assay. The newly developed MALDI-QqQ-MS/MS DBS assay as well the MALDI-QqQ-MS/MS plasma assay can therefore be used for TDM and for monitoring of the patient's compliance to the prescribed drug regimen. The easy handling to obtain DBS and the possibility to store the DBS collection cards for prolonged periods should make this sampling technique suitable for obtaining whole blood samples from HIV-1-infected children in remote areas in developing countries. We expect that DBS can be used in general for quantification of metabolites, drugs, and nutrients by MALDI-QqQ mass spectrometry.

Acknowledgements We kindly thank Abbott Laboratories for the donation of the protease inhibitor drugs lopinavir and ritonavir, and Pfizer for nelfinavir. This study was financially supported by Top Institute Pharma (project T4-212). The consortium project (T4-212) consists of the Departments of Neurology and Virology of the Erasmus MC (Rotterdam), the Departments of Pediatrics and Clinical Pharmacy of the Radboud University Nijmegen Medical Center (UMC St. Radboud), TNO-Quality of Life (Zeist), and GlaxoSmithKline (Zeist) (all from the Netherlands).

Open Access This article is distributed under the terms of the Creative Commons Attribution Noncommercial License which permits any noncommercial use, distribution, and reproduction in any medium, provided the original author(s) and source are credited.

\section{References}

1. Flexner C (1998) HIV-protease inhibitors. N Engl J Med 338:1281-1292

2. Palella FJ, Delaney KM, Moorman AC, Loveless MO, Fuhrer J, Satten GA, Aschman DJ, Holmberg SD (1998) Declining morbidity and mortality among patients with advanced human immunodeficiency virus infection. HIV Outpatient study investigators. N Engl J Med 338:853-860

3. Ernest CS 2nd, Hall SD, Jones DR (2005) Mechanism-based inactivation of CYP3A by HIV protease inhibitors. J Pharmacol Exp Ther 312:583-591

4. Lee CG, Gottesman MM, Cardarelli CO, Ramachandra M, Jeang KT, Ambudkar SV, Pastan I, Dey S (1998) HIV-1 protease inhibitors are substrates for the MDR1 multidrug transporter. Biochemistry 37:3594-3601

5. van Heeswijk RP, Veldkamp AI, Mulder JW, Meenhorst PL, Beijnen JH, Lange JM, Hoetelmans RM (2001) Saliva as an alternative body fluid for therapeutic drug monitoring of the nonnucleoside reverse transcription inhibitor nevirapine. Ther Drug Monit 23:255-258

6. ter Heine R, Rosing H, van Gorp EC, Mulder JW, van der Steeg WA, Beijnen JH, Huitema AD (2008) Quantification of protease inhibitors and non-nucleoside reverse transcriptase inhibitors in dried blood spots by liquid chromatography-triple quadrupole mass spectrometry. J Chromatogr B Analyt Technol Biomed Life Sci 867:205-212

7. Koal T, Burhenne H, Romling R, Svoboda M, Resch K, Kaever V (2005) Quantification of antiretroviral drugs in dried blood spot samples by means of liquid chromatography/tandem mass spectrometry. Rapid Commun Mass Spectrom 19:2995-3001

8. Guthrie R, Susi A (1963) A simple phenylalanine method for detecting phenylketonuria in large populations of newborn infants. Pediatrics 32:338-343

9. Malm M, Lindegardh N, Bergqvist Y (2004) Automated solidphase extraction method for the determination of piperaquine in capillary blood applied onto sampling paper by liquid chromatography. J Chromatogr B Analyt Technol Biomed Life Sci 809:43-49

10. Coombes EJ, Gamlen TR, Batstone GF, Leigh PN (1984) A phenytoin assay using dried blood spot samples suitable for domiciliary therapeutic drug monitoring. Ann Clin Biochem 21(Pt 6):519522

11. Chace DH, Kalas TA, Naylor EW (2003) Use of tandem mass spectrometry for multianalyte screening of dried blood specimens from newborns. Clin Chem 49:1797-1817

12. Pelerin H, Compain S, Duval X, Gimenez F, Benech H, Mabondzo A (2005) Development of an assay method for the detection and quantification of protease and non-nucleoside reverse transcriptase inhibitors in plasma and in peripherical blood mononuclear cells by liquid chromatography coupled with ultraviolet or tandem mass spectrometry detection. J Chromatogr B Analyt Technol Biomed Life Sci 819:47-57

13. Rentsch KM (2003) Sensitive and specific determination of eight antiretroviral agents in plasma by high-performance liquid chromatography-mass spectrometry. J Chromatogr B Analyt Technol Biomed Life Sci 788:339-350

14. Rezk NL, White NR, Jennings SH, Kashuba AD (2009) A novel LC-ESI-MS method for the simultaneous determination of etravirine, darunavir and ritonavir in human blood plasma. Talanta 79:1372-1378

15. Ehrhardt M, Mock M, Haefeli WE, Mikus G, Burhenne J (2007) Monitoring of lopinavir and ritonavir in peripheral blood mononuclear cells, plasma, and ultrafiltrate using a selective and highly sensitive LC/MS/MS assay. J Chromatogr B Analyt Technol Biomed Life Sci 850:249-258

16. Frerichs VA, DiFrancesco R, Morse GD (2003) Determination of protease inhibitors using liquid chromatography-tandem mass spectrometry. J Chromatogr B Analyt Technol Biomed Life Sci 787:393-403

17. Jung BH, Rezk NL, Bridges AS, Corbett AH, Kashuba AD (2007) Simultaneous determination of 17 antiretroviral drugs in human plasma for quantitative analysis with liquid chromatographytandem mass spectrometry. Biomed Chromatogr 21:1095-1104

18. Wang PG, Wei JS, Kim G, Chang M, El-Shourbagy T (2006) Validation and application of a high-performance liquid chromatography-tandem mass spectrometric method for simultaneous quantification of lopinavir and ritonavir in human plasma using semi-automated 96-well liquid-liquid extraction. J Chromatogr A 1130:302-307

19. van Kampen JJ, Burgers PC, Gruters RA, Osterhaus AD, de Groot R, Luider TM, Volmer DA (2008) Quantitative analysis of antiretroviral drugs in lysates of peripheral blood mononuclear cells using MALDI-triple quadrupole mass spectrometry. Anal Chem 80:4969-4975

20. van Kampen JJ, Burgers PC, de Groot R, Gruters RA, Luider TM (2009) Biomedical application of MALDI mass spectrometry for small-molecule analysis. Mass Spectrom Rev (in press)

21. van Kampen JJ, Verschuren EJ, Burgers PC, Luider TM, de Groot R, Osterhaus AD, Gruters RA (2007) Validation of an HIV-1 inactivation protocol that is compatible with intracellular drug 
analysis by mass spectrometry. J Chromatogr B Analyt Technol Biomed Life Sci 847:38-44

22. Wagner M, Varesio E, Hopfgartner G (2008) Ultra-fast quantitation of saquinavir in human plasma by matrix-assisted laser desorption/ ionization and selected reaction monitoring mode detection. J Chromatogr B Analyt Technol Biomed Life Sci 872:68-76

23. Hatsis P, Brombacher S, Corr J, Kovarik P, Volmer DA (2003) Quantitative analysis of small pharmaceutical drugs using a high repetition rate laser matrix-assisted laser/desorption ionization source. Rapid Commun Mass Spectrom 17:2303-2309

24. Sleno L, Volmer DA (2005) Some fundamental and technical aspects of the quantitative analysis of pharmaceutical drugs by matrix-assisted laser desorption/ionization mass spectrometry. Rapid Commun Mass Spectrom 19:1928-1936

25. Rathore R, Corr J, Scott G, Vollmerhaus P, Greis KD (2008) Development of an inhibitor screening platform via mass spectrometry. J Biomol Screen 13:1007-1013
26. CDER (2001) Center for Drug Evaluation and Research (CDER) guidance for industry: bioanalytical method validation. CDER, Silver Spring

27. Droste JA, Verweij-Van Wissen CP, Burger DM (2003) Simultaneous determination of the HIV drugs indinavir, amprenavir, saquinavir, ritonavir, lopinavir, nelfinavir, the nelfinavir hydroxymetabolite M8, and nevirapine in human plasma by reversed-phase highperformance liquid chromatography. Ther Drug Monit 25:393-399

28. Delaugerre C, Teglas JP, Treluyer JM, Vaz P, Jullien V, Veber F, Rouzioux C, Chaix ML, Blanche S (2004) Predictive factors of virologic success in HIV-1-infected children treated with lopinavir/ritonavir. J Acquir Immune Defic Syndr 37:1269-1275

29. Altman DG, Bland JM (1983) Measurements in medicine: the analysis of method comparison studies. The Statistician 32:307-317

30. Bland JM, Altman DG (1995) Comparing methods of measurement: why plotting difference against standard method is misleading. Lancet 346:1085-1087 